\title{
The association between fear of Covid-19 and smartphone addiction among individuals: the mediating and moderating role of cyberchondria severity
}

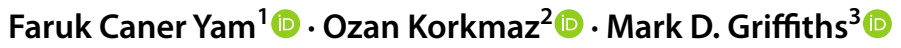 \\ Accepted: 16 September 2021 / Published online: 20 October 2021 \\ (c) The Author(s), under exclusive licence to Springer Science+Business Media, LLC, part of Springer Nature 2021
}

\begin{abstract}
The coronavirus disease-2019 (COVID-19) has quickly spread all over the world and has contributed to psychological consequences including fear of the virus. Depending upon the severity of their problems, individuals often search the internet via their mobile devices to understand whether the symptoms they perceive are symptoms of the disease. This condition has been termed 'cyberchondria'. In this context, the aim of this study is examine the mediating and moderating role of cyberchondria severity in the association between smartphone addiction and the fear of COVID-19. The sample comprised 520 participants (335 females [64.4\%], 185 males [35.6\%] aged 17 to 65 years [Mean $=28.61$ years, $\mathrm{SD}=10.60]$ ). A survey included the Cyberchondria Severity Scale Short-Form, The Smartphone Addiction Scale-Short Version, and The Fear of COVID-19 Scale. Structural equation modeling and SPSS Process Macro moderator variable analysis were used to test the research model. The study found a positive association between smartphone addiction, fear of COVID-19, and cyberchondria severity. Cyberchondria severity had both moderating and mediating role in the association between smartphone addiction and the fear of COVID-19. In conclusion, it has been determined that during the COVID-19 pandemic, cyberchondria severity has negative effects on individuals' fear of COVID-19.
\end{abstract}

Keywords Cyberchondria severity $\cdot$ Fear of COVID-19 $\cdot$ Health concern $\cdot$ Pandemic $\cdot$ Smartphone addiction

\section{Introduction}

The coronavirus disease-2019 (COVID-19) which first appeared in China in December 2019, quickly spread all over the world. The World Health Organization declared the disease as a pandemic in March 2020 (WHO, 2020a). Due to the high transmission rate of the COVID-19 virus, the number of cases and deaths seen around the world has increased rapidly. At the time of writing, the number of COVID-19 cases was over 44 million and over 1.7 million deaths (WHO, 2020b). It is accepted that the rapidly

Ozan Korkmaz

pd.ozankorkmaz@gmail.com

1 Justice Vocational School, Gazisomanpaşa University, Tokat, Turkey

2 Faculty of Education, Karamanoglu Mehmetbey University, Karaman, Turkey

3 Psychology Department, Nottingham Trent University, Nottingham, UK spreading negative effects of the COVID-19 virus is a potential death threat among individuals and societies (Pakpour \& Griffiths, 2020). Therefore, countries have implemented various measures including national lockdowns, curfews, wearing masks in public places, spatial distance practices, and travel restrictions since the very beginning of the pandemic process to reduce the negative effects of the virus (Liu et al., 2020; Xiang et al., 2020). As the destructive impact of the pandemic on the world has increased, so too has the number and frequency of these measures increased (Cao et al., 2020; Zhang \& Ma, 2020). However, these increased measures have caused individuals to radically change their daily routines and has resulted in increased fear and panic among many individuals (Huang \& Zhao, 2020; Rajkumar, 2020; Qiu et al., 2020). In addition, the intense implementation of the social isolation measure to reduce the transmission power of the virus during the pandemic process and the constant telling of individuals what they should do to protect themselves from the virus caused an increase in the fear of COVID-19 (Lau et al., 2010). Because the measures that are repeated and excessively emphasized during pandemic 
periods have a positive function such as raising awareness of the dangers of COVID-19 among individuals, it can also contribute to individuals worrying more about their health (Stacevic \& Berle, 2013). For this reason, individuals who have been exposed to intense warnings about the virus during the COVID-19 pandemic experience health concerns, constantly check for symptoms of the virus, and start doing their own online research to assure themselves (Cao et al., 2020; Gao et al., 2020). Consequently, individuals have started to feel fear and anxiety about the possibility of COVID-19 infecting them and/or their families (Asmundson \& Taylor, 2020; Pakpour \& Griffiths, 2020).

Most individuals who stayed and/or worked from home during the pandemic period have accessed developments related to COVID-19 via the internet (e.g., news on social media) (Jungmann \& Witthöft, 2020). Because in this period, individuals had to spend most of their daily time at home due to pandemic measures and started to access all developments related to the pandemic via the internet (Garcia-Priego et al., 2020). This situation caused the production and rapid spread of many false information about the COVID-19 virus in cyber environments during the pandemic period (Brown et al., 2019; Garfin et al., 2020; Laato et al., 2020a, b). However, people have started to share this information about the pandemic without evaluating whether it is correct or wrong (Hashemi et al., 2020). Because, researches state that individuals are not very careful about whether the sharing about the virus is correct during pandemic periods and they carry out an intense sharing traffic about the epidemic (Bao et al., 2020; Garfin et al., 2020). In addition, those with high levels of anxiety (Brailovskaia \& Margraf, 2021; Elhai et al., 2020a, b), low tolerance for uncertainty (He et al., 2021), fear of missing out on developments related to the disease (Casale \&Flett, 2020; Gioia et al., 2021) and individuals who feel high levels of loneliness (Gioia et al., 2021) are doing more research on the COVID-19 virus online and sharing more information online. The posts made to protect themselves, to follow the developments related to the pandemic, and to control health concerns started to get out of control after a while, and these posts in cyber environments started to cause individuals to increase their fear of COVID-19 (Jugmann et al., 2020). This sharing of misinformation in online environments can also cause an increase in individuals' fear of COVID-19 (Jugmann et al., 2020). This situation has led to the formation of a new problem area related to the COVID-19 pandemic, and has shown the risk of emotional transmission due to sharing in online environments beyond the risk of physical transmission of the virus (Gao et al., 2020; Roy et al., 2020). The World Health Organization stated that the misinformation that individuals access online concerning COVID-19 can cause psychological and physical pathological consequences (WHO, ). In various parts of the world, deaths have been reported of individuals who have followed the online advice and misinformation about COVID-19 treatment (Milliyet Newspaper, 2020; Sabah Newspaper, 2020; Euronews-Internet News, 2020). Such stories demonstrate how serious the situation can be for some individuals. Consequently, the World Health Organization has recommended that countries address the information needs of all individuals concerning COVID-19 and that accurate and reliable information is provided in all media channels in order to prevent other problems (WHO, 2020b). In fact, there have been attempts to open a special area on the WHO website to refute false information concerning COVID-19 (WHO, 2020c).

When research concerning the COVID-19 pandemic and the statements made by WHO are evaluated together, it demonstrates that it is important to not only protect individuals from the risk of physical transmission of the disease, but also to support their mental health (Ahorsu et al., 2020; Wang et al., 2020). Previous studies have also shown that individuals can experience intense negative emotions such as stress, fear, and anxiety during pandemic periods (e.g., H1N1-2010, SARS-2009) (Lau et al., 2010; Peng et al., 2010; SaadatianElahi et al., 2010). Consequently, considering the transmission rate of the COVID-19 virus, the potential risk of death, and the rate of spread throughout the world, it is inevitable that some individuals will experience fear and anxiety about this issue (Hashemi et al., 2020). In an environment that rapidly affects the whole world and thousands of new cases and deaths every day, the mental health concerns of individuals continue to increase gradually (Ahorsu et al., 2020; Fitzpatrick et al., 2020).

Many studies have reported that anxiety and stress caused by the fear of COVID-19 negatively affects the mental health of individuals (e.g., Ahorsu, et al., 2020; Bakioğlu et al., 2020; Lai et al., 2020; Xiao et al., 2020). In addition to these negative effects of the pandemic, another negative effect is the increased use of technology. This situation has made individuals vulnerable to the negative effects of the internet and online tools. Due to the measures implemented in the COVID-19 pandemic, people who stayed at home started to follow the developments related to the pandemic through online communication tools, and this caused individuals to use technological elements (internet, social media, smart phone) more (Kiraly et al., 2020; Mattiuzzi \& Lippi, 2020). For this reason, it is said that the increase in the use of internet and information technologies during the pandemic period may also affect the fears of individuals against COVID-19 (Elhai et al., 2020a, b; Hashemi et al., 2020). With this increasing use of technology, individuals are exposed to more news of COVID-19, as well as they may encounter many false information about the epidemic (Bao et al., 2020). In addition, it is reported that the intense use of technological tools (internet, social media, smart phone) 
by people causes false information about COVID-19 to spread rapidly in the society and this situation triggers health anxiety in people (Rovetta \& Bhagavathula, 2020; Xiao et al., 2020). Individuals affected by the information and news about the pandemic shared online increasingly feel anxiety, fear and anxiety (Li et al., 2020). Prior to the pandemic, studies reported that excessive use of smartphones, internet and social media were associated with anxiety, stress, and depression among a minority of individuals (Bragazzi \& Puente, 2014; Çakır \& Oğuz, 2017; Franchina et al., 2018; Soni et al., 2017). Given the apparent increase in technology use of individuals during the COVID-19 pandemic, this may also have a negative effect on some individuals' fear of COVID-19 (Gao et al., 2020; Kiraly et al., 2020).

Due to the excessive use of smartphones by some individuals, research into smartphone addiction has emerged (Griffiths \& Szabo, 2014; Kwon et al., 2013). With smartphones, individuals can engage in many activities such as accessing the internet, playing games, and accessing social media on a single device, wherever and whenever they want (Geser, 2006; Griffiths, 2013). The fact that individuals can engage in many different activities on a single device has led to a large increase in smartphone use (King et al., 2013; Yllmaz et al., 2015). During the COVID-19 pandemic, the main source of information for individuals at home has been online websites (Garcia-Priego et al., 2020). In this period, individuals had to use the internet and technological devices more in order to reach news about COVID-19 (Gao et al., 2020; Kiraly et al., 2020).

The increased use of technology and online communication tools in the COVID-19 pandemic has increased the risks of individuals becoming addicted to smartphones (Duran, 2020; Elhai et al., 2020a, b; Gür, 2020). Because individuals have started to use the internet and social media more actively, both to communicate with other people and to follow the developments related to the epidemic, due to the restricted social life during the pandemic period and the measures taken due to the pandemic (Xiao et al., 2020). Since smartphones offer individuals the opportunity to use all online communication tools through a single device (Kwon et al., 2013), the rate of smartphone use by individuals has increased gradually during the pandemic period (Elhai et al., 2020a, b; Gür, 2020). It is also stated that the use of smartphones as a means of getting emotional support and coping during the social isolation periods in the COVID-19 pandemic may cause the time spent by individuals with their smartphones to prolong (Sha et al., 2019). This result shows that smartphones are used not only as a means of obtaining information during the pandemic period, but also as a means of socialization and support seeking. Due to all these reasons explained above, it can be said that the risk of smartphone addiction is increasing, as the time spent by individuals with smartphones is gradually increasing in the COVID-19 pandemic.

Studies during the pandemic have also shown that individuals are not selective about the accuracy of sharing and information about the disease and that they constantly share information concerning COVID-19 (Bao et al., 2020; Garfin et al., 2020). As the time spent on smartphones increases, individuals are more likely to encounter false information about the pandemic and fears may rise (Lei et al., 2020; Li et al., 2020). Furthermore, intensive use of smartphones by individuals causes the rapid spread of misinformation about COVID-19 in the society and this situation causes serious increases in individuals' health concerns (Mattiuzzi \& Lippi, 2020; WHO, 2019; Wang et al., 2020). COVID-19 fears are likely to rise due to a vicious circle in which individuals turn to their smartphones, internet, and social media in order to relieve their concerns and reduce the stress they experience, but can become a negative coping method because the information they find increases their stress, anxiety and/or fear (Qiu et al., 2020; Xiao et al., 2020).

Individuals' reactions to pandemics are known to vary (White \& Horvitz, 2009). However, studies have reported that individuals experience more anxiety and fear during pandemic periods and therefore develop behaviors that will make them feel safe (Gaygisiz et al., 2012; Main et al., 2011). Depending upon the severity of the anxiety, individuals often search the internet to understand whether the symptoms they perceive are symptoms of the disease (Norr et al., 2015; White \& Horvitz, 2009). This condition, which has been termed 'cyberchondria', is defined as the behavior of individuals who experience excessive fear of being sick to search for the symptoms they perceive online (Starcevic \& Berle, 2013). In studies on cyberchondria, the main reason for this behavior is health anxiety (Norr et al., 2015; Starcevic \& Berle, 2013).

It has been found that social isolation during the COVID-19 period, the increasing use of the internet and technological devices, and the easy access to all kinds of information about the virus online may cause the increase of cyberchondria behavior during the pandemic period (Rovetta \& Bhagavathula, 2020). Because in some studies in the literature, it has been reported that there is a positive correlation between problematic internet use and cyberchondria (Starcevic, 2017; Starcevic \& Berle, 2013; Vismara et al.,2020; White \& Horvitz, 2009). This situation has caused individuals to encounter more sharing about the pandemic online. In addition, as the effect of the pandemic expanded, the health concerns of individuals began to rise and the frequency of observation of cyberchondria behaviors began to increase as the searches they made on the internet about COVID-19 increased (Husnayain et al., 2020). In order to alleviate this concern, people searching the internet have faced many wrong information about the pandemic, 
and even some wrong individuals have been harmed due to incorrect information (Kim \& Dennis, 2019). As a result, with the increase in online tool usage rates during the pandemic period, the rates of individuals exhibiting cyberchondria behaviors have also increased (Hashemi et al., 2020; Laato et al., 2020a, b).

It is stated that the risk factors of individuals becoming technology addicted increase in the COVID-19 pandemic (Király et al., 2020). In support of this opinion, some studies in the literature state that the time individuals spend with the internet, social media and smartphones during the COVID19 pandemic has increased (Gao et al., 2020; Garcia-Priego et al., 2020; Hashesmi et al., 2020; Kayiş et al., 2021). In addition, due to the social distance and social isolation measures implemented during the pandemic period, people had to spend most of their time at home (Oosterhoff et al., 2020). During this time, they stayed at home, they started to use online tools more intensively to follow the developments related to the pandemic and to communicate with their relatives, which led to an increase in the time individuals spend on the internet, social media and smart phone (Alheneidi et al., 2021; Garcia-Priego et al., 2020; Islam et al., 2020a, b; King et al., 2020). On the other hand, individuals who feel a high level of health anxiety have implemented a stricter social isolation measure and have started to meet all their communication and social needs through online communication tools (Elhai et al., 2021). With the increase in time spent with online communication tools and technological devices, individuals have encountered more news about the pandemic shared online, and as a result, they have begun to feel more health anxiety (Kayis et al., 2021; Schimmenti et al., 2020; Vismara et al., 2020). Individuals with increased health anxiety and intense use of online communication tools have tended to do more online research about the disease (Cline and Haynes, 2001; Doherty-Torstrick et al., 2016; Starcevic, 2017). These findings in the literature form the basis of a possible relationship between the smartphone, cyberchondria and fear of COVID-19 investigated in the current research. Because it can be thought that individuals who use their smartphones intensively in the COVID19 pandemic will be exposed to a lot of information about the virus, so their health concerns will increase and they will do research on the virus over the internet to control these concerns. On the other hand, the Person-Affect-Cognition-Execution (I-PACE) model developed by Brand et al. (2016) forms a basis for the relations between the variables in the current research and the established structural equation model. According to the I-PACE model, it is stated that excessive internet and smartphone use of individuals causes anxiety and stress in them, and individuals tend to use the internet again to cope with these anxiety and stress (Brand et al., 2016). In the COVID-19 pandemic, it seems very possible for individuals to enter a vicious circle expressed in the
I-PACE model, and this situation to have a negative effect on individuals' fear of COVID-19. As a result, when all the above research findings are evaluated together, it is thought that smartphone addiction may increase the cyberchondria behaviors and the fear of COVID-19, but the cyberchondria behaviors performed over the smartphone may cause individuals to increase their fear of COVID-19 more.

\section{The present study}

In a report published by We Are Social (2020a), it was claimed that among the world's 7.5 billion individuals, $60 \%$ are internet users, $67 \%$ use smartphones, and $49 \%$ are active social media users. In the same report, it was reported that the means used by individuals for internet access was smartphones with a rate of $92 \%$. This means that approximately 4.5 billion of the world population is internet users and approximately 3.8 billion are social media users. In another report, We Are Social (2020b) claimed that in Turkey which has a population of 83.88 million (where the present study was carried out), 92\% use smartphones, $64 \%$ use social media and $74 \%$ are internet users. These results suggest Turkey's internet and technology utilization rate is well above the world average. It is thought that this increase in the use of technology during the pandemic period may increase the likelihood of individuals encountering misinformation concerning COVID19 , and that this will be associated with increased levels of fear and anxiety. For all these reasons, the present study examined the mediating and moderating role of cyberchondria severity in the association between smartphone addiction and the fear of COVID-19. In the present study, the following hypotheses $\left(\mathrm{H}_{\mathrm{s}}\right)$ were examined:

$\mathrm{H}_{1}$ Smartphone addiction will positively influence the fear of COVID-19.

$\mathrm{H}_{2}$ Smartphone addiction will positively influence the severity of cyberchondria.

$\mathrm{H}_{3}$ Cyberchondira severity will positively influence the fear of COVID - 19 .

$\mathrm{H}_{4}$ Cyberchondria severity will have a mediating role in the association between smartphone addiction and the fear of COVID-19.

$\mathrm{H}_{5}$ Cyberchondria severity will have a moderating role in the association between smartphone addiction and the fear of COVID-19. 


\section{Method}

\section{Participants and procedure}

The participants comprised 520 individuals [335 females (64.4\%), 185 males (35.6\%)]. Participants' ages ranged from 17 to 65 years $($ mean $=28.61 ; \mathrm{SD}=10.60)$. A total of 520 of the participants stated that they had low socioeconomic level (10\%), 421 medium socioeconomic level (81\%), and 47 high socioeconomic level (9\%). Participants were recruited through public social media groups. The data were collected online from volunteer participants (via Google Forms in October and November 2020). The scales took approximately $10 \mathrm{~min}$ to respond.

\section{Measures}

\section{The Cyberchondria Severity Scale Short-Form (CSS-12)}

McElroy and Shevlin (2014) originally developed the 33-item Cyberchondria Severity Scale. A shorter 12-item version (CSS-12) was then developed by McElroy et al. (2019). The CSS-12 assesses the severity of individuals searching for disease symptoms online. The scale comprises four sub-dimensions (i.e., Excessiveness, Distress, Reassurance, Compulsion). Items are scored using five-point scale ranging from 1 (Never) to 5 (Always). Total scores that can be obtained from the scale is ranging from 0 to 60 . The higher total score means a high level of cyberchondria severity.

The original CSS was adapted into Turkish by Selvi et al. (2018). However, the Turkish validity and reliability study of the CSS-12 has not been conducted. Therefore, the present study tested the validity (using confirmatory factor analysis [CFA]) and reliability [Cronbach Alpha internal consistency coefficients] of the CSS-12. CFA examining the fourfactor construct validity of the scale was carried out. The goodness of fit values were: $\chi^{2}=219.01, p<0.001, \mathrm{df}=48$; $\mathrm{CFI}=0.943$; $\mathrm{TLI}=0.922$; $\mathrm{RMSEA}=0.08(90 \% \mathrm{CI}=0.07$ 0.09). Item-factor loads for the four-factor structure varied between 0.48 and 0.90 . Calculated corrected item-total correlations for the scale vary between 0.58 and 0.85 . The Cronbach Alpha internal consistency coefficient for the total score in the present study was very good (0.89) and good to very good for the sub-dimensions: Excessiveness (0.83), Distress (0.79), Reassurance (0.70), and Compulsion (0.80).

\section{The Smartphone Addiction Scale-Short Version (SAS-SV)}

The scale was developed by Kwon et al. (2013) and assesses the risk of smartphone addiction (Turkish version:
Noyan et al., 2015). It comprises 10 items and is unidimensional. Items are responded to on a six-point scale from 1 (I strongly disagree) to 6 (I strongly agree) with total scores ranging from 10 to 60 . The higher the score, the higher the risk of smartphone addiction. The Cronbach Alpha coefficient in the present study was excellent (0.92).

\section{The Fear of COVID-19 Scale (FCV-19S)}

The scale was developed by Ahorsu et al. (2020) and assesses fear of COVID-19. It was validated by Satici et al. (2020). It comprises seven items and is uni-dimensional. Items are responded to on a five-point scale from 1 (Strongly disagree) to 5 (Strongly agree) with total scores ranging from 7 to 35 . The higher the score the higher the level of fear of COVID-19. The Cronbach Alpha coefficient was found to be sufficient in this study $(0.86)$.

\section{Data analysis}

Before the analysis, the distribution of the data was examined. When the kurtosis and skewness values were examined, the data showed a normal distribution Pearson correlation, the independent samples t-test and one-way ANOVA analysis were used. Structural equation modeling and SPSS Process Macro moderator variable analysis were used to test the research model (Fig. 1).

In the examination of the goodness of fit values of the structural equation model, $\chi^{2} / \mathrm{df}<5$; GFI, TLI, CFI, $>0.90$; RMSEA $<0.10$ values were taken as basis for good fits (Hu \& Bentler, 1999). Bootstrapping tests were used to examine whether cyberchondria severity mediated the relationship between the smartphone addiction and the fear of COVID-19 (Hayes, 2018). To test the indirect effect of smartphone addiction on fear of COVID-19, bootstrapping confidence interval was calculated (10.000 resamples). IBM SPSS Statistics 26 and AMOS Graphics 24 were used for the analyses.

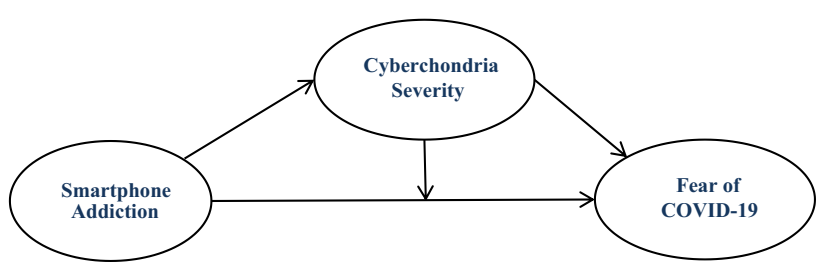

Fig. 1 Research model 


\section{Results}

\section{Descriptive statistics}

Information on the descriptive statistics of the participants is shown in Table 1. According to the results of the independent samples t-test, females had a higher fear of COVID-19 than males $\left(\mathrm{t}_{(518)}=3.67, p<0.001\right)$. However, there were no gender differences for smartphone addiction and cyberchondria severity. According to a one-way ANOVA, fear of COVID-19, smartphone addiction and cyberchondria severity did not differ according to the socioeconomic levels of the participants. As expected, in relation to the duration of daily smartphone use, the smartphone addiction levels of the participants differed significantly in all groups $\left(\mathrm{F}_{(517,2)}=63.69, p<0.001\right)$. However, fear of COVID-19 and cyberchondria severity did not differ according to duration of daily smartphone use. In relation to duration of daily social media use time, levels of smartphone addiction $\left(\mathrm{F}_{(517,2)}=1.04, p<0.001\right)$ and cyberchondria severity $\left(\mathrm{F}_{(517,2)}=5.91, p<0.001\right)$ significantly differed. There was a significant difference in all three groups in relation to duration of daily social media use time ( $0-1$ h daily use, $1-3 \mathrm{~h}$ daily use, above $3 \mathrm{~h}$ daily use) in smartphone addiction. However, it was found that individuals who spent more than three hours daily on social media had higher levels of cyberchondria than those who spent less daily time on social media. Finally, it was found that fear of COVID-19 did not differ according to daily time spent on social media use.

\section{Correlation analysis}

Correlations between variables are shown in Table 2. As shown in Table 2, the variables examined in the study had significant positive relationships. Fear of COVID-19 had a significant relationship with cyberchondria and smartphone addiction. In addition, there was a positive significant relationship between cyberchondria and smartphone addiction.

Table 1 Descriptive statistics

\begin{tabular}{|c|c|c|c|c|c|c|c|c|}
\hline Variables & $f$ & $\%$ & $\mathrm{M}_{1}$ & $\mathrm{SD}_{1}$ & $\mathrm{M}_{2}$ & $\mathrm{SD}_{2}$ & $\mathrm{M}_{3}$ & $\mathrm{SD}_{3}$ \\
\hline \multicolumn{9}{|l|}{ Gender } \\
\hline Male & 335 & 64.4 & 15.92 & 5.95 & 31.49 & 9.70 & 28.44 & 12.36 \\
\hline Female & 185 & 35.6 & 13.99 & 5.59 & 31.41 & 9.73 & 26.38 & 11.63 \\
\hline \multicolumn{9}{|c|}{ Socioeconomic status } \\
\hline Low & 52 & 10 & 16.77 & 5.53 & 33.94 & 9.67 & 28.79 & 13.22 \\
\hline Middle & 421 & 81 & 15.07 & 5.69 & 31.33 & 9.62 & 27.45 & 11.90 \\
\hline High & 47 & 9 & 15.26 & 7.70 & 29.87 & 10.10 & 28.83 & 13.08 \\
\hline \multicolumn{9}{|c|}{ Daily smartphone use } \\
\hline $0-1 \mathrm{~h}$ & 44 & 8.25 & 14.57 & 6.37 & 30.16 & 10.08 & 17.25 & 9.37 \\
\hline $1-3 \mathrm{~h}$ & 196 & 37.7 & 14.89 & 5.43 & 30.83 & 9.15 & 23.24 & 9.31 \\
\hline Above $3 \mathrm{~h}$ & 280 & 53.8 & 15.62 & 6.12 & 32.11 & 9.99 & 32.48 & 12.07 \\
\hline \multicolumn{9}{|c|}{ Daily social media use } \\
\hline $0-1 \mathrm{~h}$ & 141 & 27.1 & 14.65 & 5.53 & 29.75 & 10.11 & 20.10 & 9.56 \\
\hline $1-3 \mathrm{~h}$ & 220 & 42.3 & 15.51 & 5.78 & 31.10 & 9.50 & 26.78 & 10.59 \\
\hline Above $3 \mathrm{~h}$ & 159 & 30.6 & 15.44 & 6.35 & 33.48 & 9.29 & 35.74 & 11.38 \\
\hline
\end{tabular}

$\mathrm{M}_{1,2,3}$ : Mean, $\mathrm{SD}_{1,2,3}$ : Standard Deviation

$\mathrm{M}_{1}$ and $\mathrm{SD}_{1}$ : Fear of COVID-19, $\mathrm{M}_{2}$ and $\mathrm{SD}_{2}$ : Cyberchondria Severity, $\mathrm{M}_{3}$ and $\mathrm{SD}_{3}$ : Smartphone Addiction

Table 2 Inter-correlations of the variables and descriptive statistics

\begin{tabular}{llllllll}
\hline Variables & 1 & 2 & 3 & Mean & SD & Skewness & Kurtosis \\
\hline 1 Fear of COVID-19 & 1.00 & & & 15.26 & 5.90 & 0.70 & -0.02 \\
2 Cyberchondria severity & $0.31^{* * *}$ & 1.00 & & 31.46 & 9.70 & 0.04 & -0.45 \\
3 Smartphone addiction & $0.28^{* * *}$ & $0.37^{* * *}$ & 1.00 & 27.71 & 5.90 & .46 & -0.58 \\
\hline
\end{tabular}

$* * * p<0.001$ 
Fig. 2 Mediation model of the relationships between the research variables

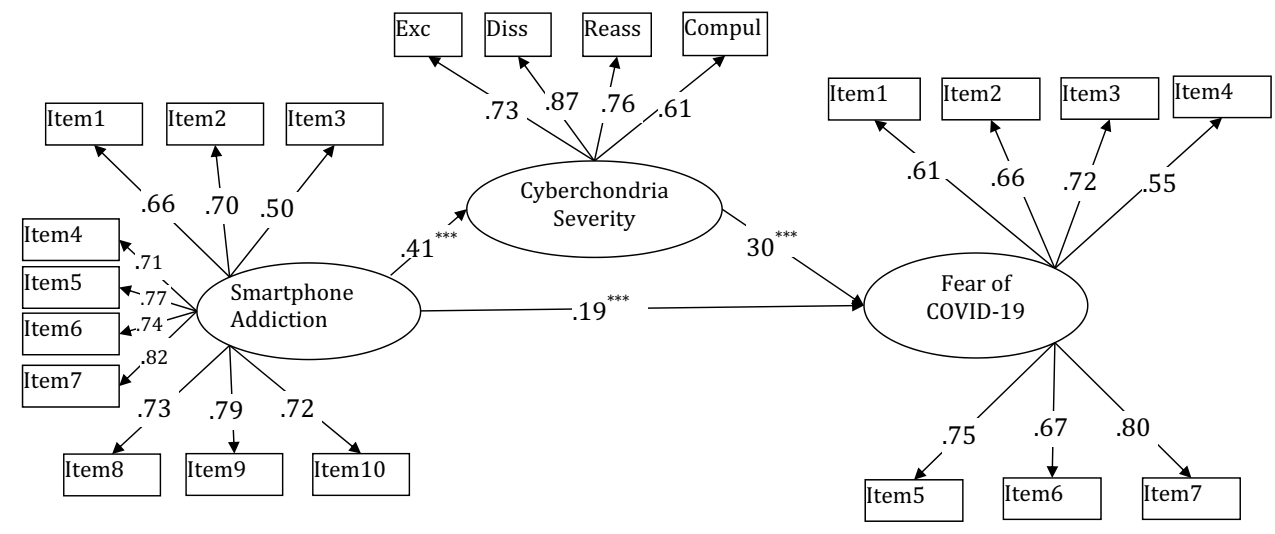

Table 3 Bootstrapping analysis results of the model

\begin{tabular}{llll}
\hline Pathway & Coefficient & Lower-bound & Upper-bound \\
\hline Direct effect & & & \\
$\mathrm{SA} \rightarrow$ FCV19 & 0.19 & 0.077 & 0.308 \\
$\mathrm{SA} \rightarrow$ CS & 0.41 & 0.311 & 0.498 \\
$\mathrm{CS} \rightarrow$ FCV19 & 0.30 & 0.186 & 0.425 \\
Indirect effect & & & \\
$\mathrm{SA} \rightarrow \mathrm{CS} \rightarrow$ FCV19 & 0.12 & 0.074 & 0.185 \\
\hline
\end{tabular}

$\mathrm{R}=0.42, \mathrm{R}^{2}=0.18, N=520$

CS cyberchondria severity; FCV19 fear of COVID-19; SA smartphone addiction

\section{Mediating role of cyberchondria in the association between smartphone addiction and fear of COVID-19}

The mediating role of cyberchondria severity in the association between smartphone addiction and fear of COVID19 was tested. The figure containing the findings of the research model is shown in Fig. 2. Analysis demonstrated that the goodness of fit values of the model were within acceptable limits $\left[\chi^{2}=580.534, \mathrm{df}=181, p<0.001\right.$, $\chi^{2} / \mathrm{df}=3.207 ; \mathrm{GFI}=0.90 ; \mathrm{TLI}=0.92 ; \mathrm{CFI}=0.94$; RMSEA $=0.07(90 \% \mathrm{CI}=0.06-0.07)]$. Looking at the direct effects in the model were examined, it was found that smartphone addiction predicted fear of COVID-19 $(\beta=0.19, p<0.001)$ and cyberchondria severity $(\beta=0.41$, $p<0.001)$. Similarly, cyberchondria severity predicted fear of COVID-19 positively $(\beta=0.30, p<0.001)$. When the findings regarding the indirect effects in the model were examined, it was found that smartphone addiction predicted fear of COVID-19 via cyberchondria severity $(\beta=0.12, p<0.001)$. Path coefficients for indirect and direct effects are shown in Table 3.

As can be seen in Table 3, the path coefficient of all direct and indirect effects in the model were significant.
Table 4 Analysis findings regarding moderating impact

\begin{tabular}{lllrll}
\hline Variables & Coefficient & SE & \multicolumn{1}{l}{$t$} & \multicolumn{1}{l}{ LLCI } & ULCI \\
\hline Constant & 15.26 & 0.27 & 56.08 & 14.726 & 15.795 \\
SA & $0.11^{* * *}$ & 0.02 & 4.73 & 0.0628 & 0.1521 \\
CS & $0.16^{* * *}$ & 0.03 & 5.83 & 0.1091 & 0.2202 \\
SA x CS & $0.01 * *$ & 0.002 & 2.59 & 0.0012 & 0.0090
\end{tabular}

$C S$ cyberchondria severity, $S A$ smartphone addiction

${ }^{* *} p<0.01, * * * p<0.001, \mathrm{R}=0.42, \mathrm{R}^{2}=0.18, N=520$

Table 5 The effects of smartphone addiction on fear of COVID-19 for different levels of cyberchondria severity

\begin{tabular}{llllll}
\hline Levels & Coefficient & SE & $T$ & LLCI & ULCI \\
\hline Low & 0.06 & 0.03 & 1.85 & -0.0035 & 0.1178 \\
Middle & $0.11^{* * *}$ & 0.02 & 4.73 & 0.0628 & 0.1521 \\
High & $0.16^{* * *}$ & 0.03 & 5.46 & 0.1009 & 0.2145 \\
\hline
\end{tabular}

$* * * p<.001$

\section{Moderating role of cyberchondria severity in the association between smartphone addiction and fear of COVID-19}

Regression analysis was performed using Process Macro developed by Hayes (2018) in order to test the moderating role of cyberchondria severity in the effect of individuals' smartphone addiction on their fear of COVID-19. The findings of the analysis are shown in Table 4. As shown in Table 4, all variables included in the analysis explained approximately $18 \%$ of fear of COVID-19 $(\mathrm{F}=32.32$, $\left.\mathrm{R}^{2}=0.18, p<0.001\right)$. Smartphone addiction $(\beta=0.11$, $p<0.001)$ and cyberchondria severity $(\beta=0.16, p<0.001)$ had positive and significant effects on fear of COVID-19. The moderating role of cyberchondria severity was significant in the relationship between smartphone addiction and fear of COVID-19 $(\beta=0.01, p<0.01)$. It was also found that 
the increase in $\mathrm{R}^{2}$ was significant by adding the interaction between smartphone addiction and cyberchondria severity (SA x CS) to the model $\left(\mathrm{F}=6.72, \mathrm{R}^{2}\right.$ change $=0.012$, $p<0.01)$. The effects of smartphone addiction on fear of COVID-19 for different levels of cyberchondria severity are given in Table 5.

The analysis indicated that the effect of smartphone addiction on fear of COVID-19 for middle and high levels of cyberchondria severity was significant. However, it appears that this effect was not significant for low levels of cyberchondria severity. The slope graphs regarding the effects of the moderator variable are presented in Fig. 3. This shows that the effect of smartphone addiction on fear of COVID19 increases in the middle and high levels of cyberchondria severity. In the case of high cyberchondria severity, this relationship is even stronger. Therefore, if cyberchondria severity is high, the effect of smartphone addiction on fear of COVID-19 is greater. This finding indicates that cyberchondria severity is a moderator in the relationship between smartphone addiction and fear of COVID-19.

\section{Discussion}

According to the first finding of the study, there is a positive association between smartphone addiction and the fear of COVID-19. In other words, it can be said that there is a linear relationship between smartphone addiction and the fear of COVID-19. These results show that the $\mathrm{H}_{1}$ was supported. This result can be interpreted as individuals are exposed to a lot of information about the COVID-19 virus because they use their smartphones intensively during the pandemic period, and therefore their fear of COVID-19 has increased. There are studies reporting similar results in the literature. For example, Boursier et al. (2020) in their research concluded that the use of social media by individuals increased during the COVID-19 period, and this use positively predicted the fear of COVID-19 in a meaningful way. Elhai et al. (2020a) examined the relationship between problematic smartphone use and COVID-19 anxiety and found that there was a positive relationship between COVID-19 anxiety and smartphone addiction, and that smartphone addiction had a mediating effect between COVID-19 anxiety and general anxiety. Similarly, in another study, they concluded that there is a positive relationship between fear of COVID-19 and smartphone addiction (Kayiş et al., 2021). Servidio et al. (2021), it was revealed that there is a positive relationship between internet addiction and fear of COVID-19. On the other hand, in some studies, it is reported that individuals use technological devices more in the COVID-19 pandemic and this has a negative effect on their fear of COVID-19 (Hashemi et al., 2020; Kiraly et al., 2020; Yang et al., 2020). In addition, it is stated that many false information about the COVID-19 pandemic is shared in the cyber environment and the fear of COVID-19 of individuals who are exposed to them increases (Brown et al., 2019; Garfin et al., 2020). It has even been claimed that during the pandemic period, sharing misinformation concerning COVID-19 increases the risk of emotional transmission among individuals and therefore individuals start to feel anxiety and fear about the COVID-19 (Gao et al., 2020; Roy et al., 2020; WHO, 2020c).

However, with social isolation, many individuals follow all developments related to the COVID-19 pandemic via their smartphones, and it has been claimed that COVID-19 fears may rise because this causes individuals to view many inaccurate shares and news stories (Hashemi et al., 2020). In addition, it has been claimed that during the COVID-19 pandemic, there has been a large increase in internet and social media use rates among individuals (Gao et al., 2020; Jungmann \& Witthöft, 2020). In some studies, it has been reported that individuals with internet and social media addiction have higher fear of COVID-19 (Ahmad \& Murad, 2020; Garcia-Priego et al., 2020; Islam et al., 2020a, b; Yu
Fig. 3 The effects of smartphone addiction on fear of COVID-19 for different levels of cyberchondria severity

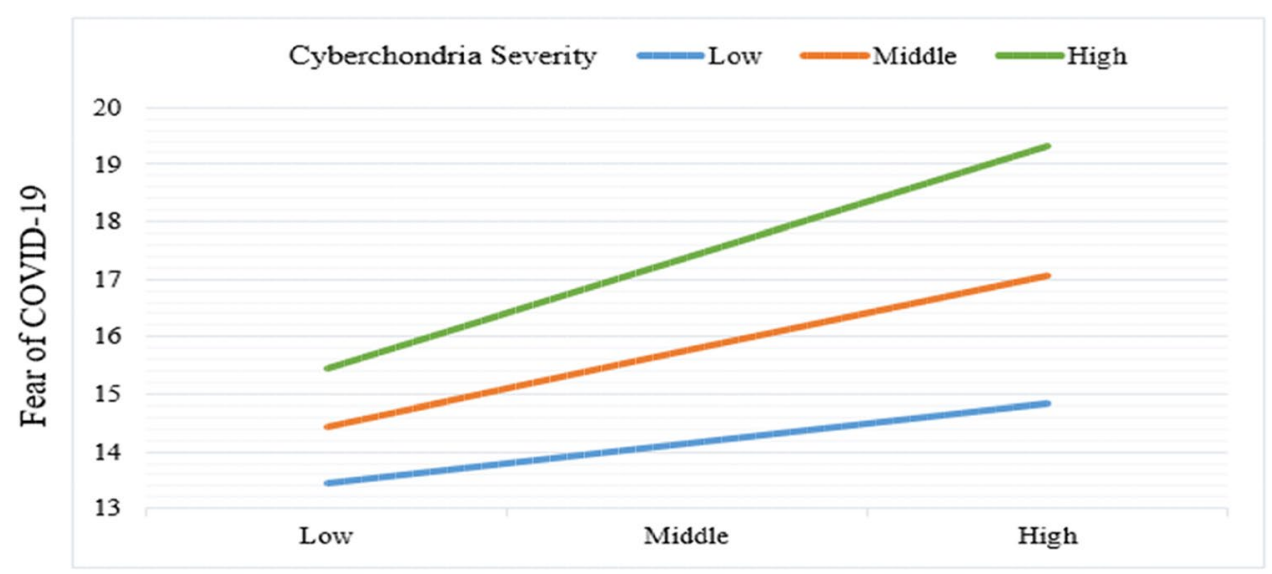

Smartphone Addiction 
et al., 2020). These studies findings concur with the present study's findings. However, the present findings are similar to the results of the studies conducted before the pandemic on smartphone addiction. Such studies have shown that smartphone addiction is associated with stress, anxiety, fear, and depression in individuals (Bragazzi \& Puente, 2014; Çakır \& Oğuz, 2017; Elhai et al., 2017, 2020b; Franchina et al., 2018; Soni et al., 2017). Taken together, the findings suggest that technological addictions have had negative effects on individuals' fear of COVID-19 during the pandemic period. In addition, since the intensive use of smartphones increases the possibility of encountering misinformation about the COVID-19 virus, this provides a possible reason for why individuals have increased fear of COVID-19.

The second finding of the study showed that there was a significant positive relationship between smartphone addiction and cyberchondria severity. This suggests that individuals with smartphone addiction during the pandemic period exhibited higher cyberchondria behaviors. This finding supported $\mathrm{H}_{2}$. There are previous studies that have reported similar results to this finding. Abdelsattar et al. (2021) in their research on a sample group of Arab individuals, concluded that there is a positive relationship between the severity of cybercoderia and fear of COVID-19. In some studies, it has been concluded that addictions to smartphones, the internet and/or social media is associated with cyberchondria severity (Fergus \& Spada, 2017; Ivanova, 2013; Ivanova \& Karabeova, 2014; Khazaal et al. 2020). Other studies have reported that individuals who experience intense anxiety and fear during pandemics engage in behaviors that make them safe (Gaygisiz et al., 2012; Main et al., 2011). Due to the measures implemented in the COVID-19 pandemic which can result in social isolation, individuals have come to follow the developments related to COVID-19 mostly on the internet via their smartphones in order to cope with their anxiety and fear and to feel safe (Duran, 2020; Elhai et al., 2020a; Gür, 2020). This may lead to an increase in technological addiction rates among individuals during the pandemic period (Elhai et al., 2020a; Gao et al., 2020). Cyberchondria severity may increase among individuals who seek out all kinds of information concerning COVID-19 during the pandemic period (Rovetta \& Bhagavathula, 2020; Sha et al., 2019). Similarly, in another study, it was concluded that individuals who were addicted to smartphones during the COVID-19 period felt more health anxiety (Elhai et al., 2021). However, with the increase of the threatening effect of the pandemic, the fact that individuals seek out more information concerning COVID-19 on the internet is a factor that may cause an increase in cyberchondria severity (Husnayain et al., 2020). In addition, in some studies have reported that the COVID-19 pandemic has contributed to an increase in the health concerns among individuals, and as a result of this, individuals tend to seek out more information about the virus on the internet which triggers cyberchondria severity (Hashemi et al., 2020; Jungmann \& Witthöft, 2020). The finding concerning the relationship between smartphone and cyberchondria severity is also consistent with the I-PACE (Interaction of Person-Affect-CognitionExecution Model) model proposed (Brand et al., 2016). In this model, it states that individuals tend to use the internet and smartphones excessively to manage mood states such as anxiety and fears. Similarly, another study found that cyberchondria severity was associated with technological addictions (Vismara et al., 2020).

The third main finding of the study found a positive relationship between cyberchondria severity and the fear of Covid 19. This result indicates that individuals with high levels of cyberchondira behavior during the pandemic period also have higher fear of COVID-19. $\mathrm{H}_{3}$ was therefore supported. This has been found in previous studies. Farooq et al. (2020) concluded that individuals who conducted a lot of research concerning COVID-19 online had high concerns about the virus. In another study, it was observed that the cyberchondria behaviors exhibited in the COVID-19 pandemic increased their fear of COVID-19 (Jungman and Witth, 2020). However, when the literature is examined, it is found that the most important determinant of cyberchondria severity is health anxiety (Norr et al. 2015; Starcevic $\&$ Berle, 2013). In some studies, it has been revealed that individuals who have health concerns in the COVID-19 pandemic tend to do intensive research on the virus on the internet in order to secure themselves (Cao et al., 2020; Gao et al., 2020). In another study, it was concluded that there was a positive relationship between the severity of individuals' cyberchondria and their fear of COVID-19 (Wu et al, 2021). Jokic-Begic et al. (2020) In a study they conducted on Croatian individuals, it was determined that as the level of cyberchondria of individuals increased, their fear of COVID-19 increased. Consequently, individuals have been found to increase their fear and anxiety levels regarding the disease because they view a lot of unconfirmed information about the virus (Laato et al., 2020a, b; Starcevic et al., 2021; Xie et al., 2011). It has been found that individuals with high cyberchondria severity seek out more information about diseases on the internet. These individuals are more likely to encounter false information about the virus because they make more internet searches concerning COVID-19.

The fourth finding of the study showed that cyberchondria severity had a partial mediating role in the association between smartphone addiction and the fear of COVID-19. This suggests that the fear of COVID-19 among individuals with smartphone addiction increases, but with high cyberchondria severity, the fear of COVID-19 among individuals increases even more. This finding supports $\mathrm{H}_{4}$. The fifth finding of the study showed that cyberchondria severity played a moderating role in the association between 
smartphone addiction and the fear of COVID-19. According to this result, it appears that the relationship between smartphone addiction and the fear of COVID-19 in cases with moderate and high levels of cyberchondria severity is stronger than in cases with low cyberchondria severity. In other words, cyberchondria severity has a significant role in the association between smartphone addiction and the fear of COVID-19. This result supports $\mathrm{H}_{5}$. In the literature, there are studies that have reached similar results regarding the relationship between smartphone addiction, cyberchondria severity and fear of COVID-19 (Ahmad \&Murad, 2020; Brown, et al., 2019; Elhai et al., 2020a; Farooq et al., 2020; Garfin et al., 2020; Husnayain et al., 2020; Jungmann \& Witthöft, 2020). On the other hand, the literature shows that fear anxiety increases during pandemic periods, and that individuals' information needs about many areas, especially health, increase and therefore tend to seek out more information online (Lau et al., 2010; Saadatian et al., 2010). In addition, it is stated that individuals exhibit more cyberchondriac behaviors during epidemic periods that affect the whole world such as a pandemic (Zheng et al., 2020). Because, with measures resulting in social isolation to prevent the spread of the virus, the time individuals spend on the internet and technological devices have increased. It can be stated that all these explanations support the present research findings.

\section{Limitations and future research}

The present study has some limitations. The study sample comprised individuals living in Turkey. Therefore, caution should be exercised when generalizing the present research results to individuals outside of the Turkish society. In future research, the present study could be repeated with groups of individuals from different countries and cultures to confirm the explanatory power of the model in other contexts. In addition, the present study was cross-sectional study and therefore is unable to provide unequivocal evidence for causality among the variables studied. Furthermore, all data were obtained by a self-report method. Therefore, longitudinal research utilizing other methodologies is needed to overcome these shortcomings. Finally, in the research model, only the relationship between smartphone addiction, cyberchondria severity and fear of COVID-19 was examined. In order to ensure the validity of the research results after the pandemic, the model can be examined with different types of fear that may affect variables such as cyberchondria and smartphone addiction.

\section{Conclusion}

The present study found that smartphone addiction was associated with an increase in COVID-19 fear among individuals during the pandemic. Similarly, during the pandemic period, it was found that individuals with higher smartphone addiction scores presumably access their smartphones to seek out health information. In addition, the study found that cyberchondria severity was associated with individuals' fear of COVID-19. In other words, during the COVID-19 pandemic, it appears that individuals who searched online for health information and were exposed to a lot of inaccurate information increased their fear of COVID-19. However, it has been observed that cyberchondria severity has both a mediating and a moderating role in the relationship between smartphone addiction and the fear of COVID-19. This research revealed that in addition to the global fear of the COVID-19 pandemic, individuals who use excessive phones and have high cyberchondria behaviors have higher fear of COVID-19. The findings here indicate that it is important to control the cyberchondria behaviors with the use of smartphones so that individuals can combat the fear of COVID-19 more effectively.

These results obtained in the research also revealed the fact that it is very important for individuals to be made aware of the negative effects of internet and information technologies during epidemic periods. For this reason, it is important for governments to utilize their relevant departments (e.g., Ministries of Health) effectively in order to provide reliable information for individuals regarding COVID-19 during the pandemic period. Consequently, individuals' anxieties and fears regarding COVID-19 would be supported in becoming more manageable. On the other hand, the findings of this research revealed that the intensive use of smartphones and research on the disease through smartphones may cause individuals to increase their fear of COVID-19 and to use their smartphones again. This situation indicates that individuals may enter a vicious circle that requires clinical intervention in terms of fear of COVID-19. For this reason, these results have revealed that it is important to examine the use of smartphones and the activities of individuals seeking psychological help due to fear of COVID-19, searching for information about diseases on their smartphones. In other words, we anticipate that, firstly, regulating the habits of using online communication tools and secondly, taking measures to reduce repetitive online disease researches can be two important strategies in order to control the COVID19 fears of individuals during the pandemic period. In addition, this research has also shown that individuals' cyberchondria behaviors play a role in increasing their fear of COVID-19. This result reveals that cyberchondria can cause a new medical crisis during epidemic periods such as pandemics, and therefore it is important to produce policies to raise awareness of individuals on how to use online tools in crisis periods such as epidemics. 
Funding The authors received no financial support for the research, authorship, and/or publication of this paper.

Data availability The datasets generated during and analyzed during the current study are available from the corresponding author on reasonable request.

\section{Declarations}

Ethical approval All procedures in this study involving human participants were conducted in accordance with the ethical standards of the 1975 Helsinki Declaration and was approved by the research team's university ethics committee.

Informed consent Informed consent for participation was obtained before completion of the survey.

Conflicts of interest The authors declare that they have no conflicts of interests.

\section{References}

Abdelsattar, M., Derar, E., Salem, A. A., \& Al-Mujaim, F. (2021). Cyberchondria severity, health anxiety, and health locus of control: The mediation role of covid-19 anxiety. Asean Journal of Psychiatry, 22(2), 1-11.

Ahmad, A. R., \& Murad, H. R. (2020). The impact of social media on panic during the COVID-19 pandemic in Iraqi Kurdistan: Online questionnaire study. Journal of Medical Internet Research, 22(5), e19556. https://doi.org/10.2196/19556

Ahorsu, D. K., Lin, C-Y., Imani, V., Saffari, M., Griffiths, M. D., \& Pakpour, A. H. (2020). The fear of COVID-19 scale: Development and initial validation. International Journal of Mental Health and Addiction. Retrieved March 30, 2020 from: https:// link.springer.com/content/pdf/10.1007/s11469-020-00270-8.pdf

Alheneidi, H., AlSumait, L., AlSumait, D., \& Smith, A. P. (2021). Loneliness and problematic internet use during COVID-19 lockdown. Behavioral Sciences, 11(1), 5. https://doi.org/10.3390/ bs11010005

Asmundson, G. J. G., \& Taylor, S. (2020). How health anxiety influences responses to viral outbreaks like COVID-19: What all decision-makers, health authorities, and health care professionals need to know. Journal of Anxiety Disorders, 71, 102211. https:// doi.org/10.1016/j.janxdis.2020.102211

Bakioğlu, F., Korkmaz, O., \& Ercan, H. (2020). Fear of COVID-19 and positivity: Mediating role of intolerance of uncertainty depression anxiety and stress. International Journal of Mental Health and Addiction. https://doi.org/10.1007/s11469-020-00331-y

Bao, Y., Sun, Y., Meng, S., Shi, J., \& Lu, L. (2020). 2019-nCoV epidemic: Address mental health care to empower society. The Lancet, 395(10224), e37-e38. https://doi.org/10.1016/S01406736(20)30309-3

Boursier, V., Gioia, F., Musetti, A., \& Schimmenti, A. (2020). Facing loneliness and anxiety during the COVID-19 isolation: The role of excessive social media use in a sample of Italian adults. Frontiers in Psychiatry, 11, 586222. https://doi.org/10.3389/ fpsyt.2020.586222

Bragazzi, N. L., \& Del Puente, G. (2014). A proposal for including nomophobia in the new DSM-V. Psychology Research and Behavior Management, 7, 155. https://doi.org/10.2147/PRBM. S41386
Brailovskaia, J., \& Margraf, J. (2021). The relationship between burden caused by coronavirus (COVID-19), addictive social media use, sense of control and anxiety. Computers in Human Behavior, 119, 106720. https://doi.org/10.1016/j.chb.2021.106720

Brand, M., Young, K. S., Laier, C., Wölfling, K., \& Potenza, M. N. (2016). Integrating psychological and neurobiological considerations regarding the development and maintenance of specific Internet-use disorders: An Interaction of PersonAffect-Cognition-Execution (I-PACE) model. Neuroscience \& Biobehavioral Reviews, 71, 252-266. https://doi.org/10.1016/j. neubiorev.2016.08.033

Brown, R. J., Skelly, N., \& Chew-Graham, C. A. (2019). Online health research and health anxiety: A systematic review and conceptual integration. Clinical Psychology: Science and Practice, 9(2), 85. https://doi.org/10.1111/cpsp.12299

Çakır, Ö., \& Oğuz, E. (2017). The correlation between high school students' loneliness levels and smart phone addiction. Mersin University Journal of the Faculty of Education, 13(1), 418-429. https://doi.org/10.17860/mersinefd.290711

Cao, W., Fang, Z., Hou, G., Han, M., Xu, X., Dong, J., \& Zheng, J. (2020). The psychological impact of the COVID-19 epidemic on college students in China. Psychiatry Research. https://doi.org/ 10.1016/j.psychres.2020.112934

Casale, S., \& Flett, G. L. (2020). Interpersonally-based fears during the COVID-19 pandemic: Reflections on the fear of missing out and the fear of not mattering constructs. Clinical Neuropsychiatry, 17(2), 88-93. https://doi.org/10.36131/CN20200211

Cline, R. J., \& Haynes, K. M. (2001). Consumer health information seeking on the Internet: The state of the art. Health Education Research, 16(6), 671-692. https://doi.org/10.1093/her/16.6.671

Doherty-Torstrick, E. R., Walton, K. E., \& Fallon, B. A. (2016). Cyberchondria: Parsing health anxiety from online behavior. Psychosomatics, 57(4), 390-400. https://doi.org/10.1016/j.psym.2016. 02.002

Duran, Y. (2020). The coronavirus triggered digital addiction. Retrieved December 7, 2020 from https://yesilay.org.tr/tr/makal eler/koronavirus-dijital-bagimligi-tetikledi

Elhai, J. D., Levine, J. C., Dvorak, R. D., \& Hall, B. J. (2017). Nonsocial features of smartphone use are most related to depression, anxiety and problematic smartphone use. Computers in Human Behavior, 69, 75-82. https://doi.org/10.1016/j.chb.2016.12.023

Elhai, J. D., Yang, H., McKay, D., \& Asmundson, G. J. (2020a). COVID-19 anxiety symptoms associated with problematic smartphone use severity in Chinese adults. Journal of Affective Disorders, 274, 576-582. https://doi.org/10.1016/j.jad.2020.05. 080

Elhai, J. D., Gallinari, E. F., Rozgonjuk, D., \& Yang, H. (2020b). Depression, anxiety and fear of missing out as correlates of social, non-social and problematic smartphone use. Addictive Behaviors, 105, 106335. https://doi.org/10.1016/j.addbeh.2020. 106335

Elhai, J. D., McKay, D., Yang, H., Minaya, C., Montag, C., \& Asmundson, G. J. (2021). Health anxiety related to problematic smartphone use and gaming disorder severity during COVID-19: Fear of missing out as a mediator. Human Behavior and Emerging Technologies, 3(1), 137-146. https://doi.org/10.1002/hbe2.227

Eueronews. (2020). Coronavirus posts on social media: 10 false facts. April 3. Retrieved January 11, 2020, from https://tr.euronews. com/2020/04/03/sosyal-medyada-koronavirus-paylasimlaridogru-bilinen-10-yanlis-corona-virus-covid-19

Farooq, A., Laato, S., \& Islam, A. N. (2020). Impact of online information on self-isolation intention during the COVID-19 pandemic: Cross-sectional study. Journal of Medical Internet Research, 22(5), e19128. https://doi.org/10.2196/19128

Fergus, T. A., \& Spada, M. M. (2017). Cyberchondria: Examining relations with problematic Internet use and metacognitive beliefs. 
Clinical Psychology \& Psychotherapy, 24(6), 1322-1330. https:// doi.org/10.1002/cpp.2102

Fitzpatrick, K. M., Harris, C., \& Drawve, G. (2020). Fear of COVID-19 and the mental health consequences in America. Psychological Trauma: Theory, Research, Pactice, and Policy, 12(1), 17-21. https://doi.org/10.1037/tra0000924

Franchina, V., Vanden Abeele, M., van Rooij, A., Lo Coco, G., \& De Marez, L. (2018). Fear of missing out as a predictor of problematic social media use and phubbing behavior among Flemish adolescents. International Journal of Environmental Research and Public Health, 15(10), 2319. https://doi.org/10.3390/ijerp h15102319

Gao, J., et al. (2020). Mental health problems and social media exposure during COVID-19 outbreak. PLOS ONE, 15(4), e0231924. https://doi.org/10.1371/journal.pone.0231924

Garcia-Priego, B. A., et al. (2020). Anxiety, depression, attitudes, and internet addiction during the initial phase of the 2019 coronavirus disease (COVID-19) epidemic: A cross-sectional study in Mexico. medRxiv. https://doi.org/10.1101/2020.05.10.20095844

Garfin, D. R., Silver, R. C., \& Holman, E. A. (2020). The novel coronavirus (COVID-2019) outbreak: Amplification of public health consequences by media exposure. Health Psychology, 39(5), 355-357. https://doi.org/10.1037/hea0000875

Gaygisiz, U., Gaygisiz, E., Ozkan, T., \& Lajunen, T. (2012). Individual differences in behavioral reactions to H1N1 during a later stage of the epidemic. Journal of Infection and Public Health, 5(1), 9-21. https://doi.org/10.1016/j.jiph.2011.09.008

Geser, H. (2006). Is the cell phone undermining the social order?: Understanding mobile technology from a sociological perspective. Knowledge, Technology \& Policy, 19(1), 8-18.

Gioia, F., Fioravanti, G., Casale, S., \& Boursier, V. (2021). The effects of the fear of missing out on people's social networking sites use during the COVID-19 pandemic: The mediating role of online relational closeness and individuals' online communication attitude. Frontiers in Psychiatry, 12, 146. https://doi.org/10.3389/ fpsyt.2021.620442

Gonçalves, A. P., Zuanazzi, A. C., Salvador, A. P., Jaloto, A., Pianowski, G., \& Carvalho, L. F. (2020). Preliminary findings on the associations between mental health indicators and social isolation during the COVID-19 pandemic. Archives of Psychiatry and Psychotherapy, 22(2), 10-19. https://doi.org/10.12740/ APP/122576

Griffiths, M. D. (2013). Social networking addiction: Emerging themes and issues. Journal of Addiction Research \& Therapy, 4(5). https://doi.org/10.4172/2155-6105.1000e118

Griffiths, M. D., \& Szabo, A. (2014). Is excessive online usage a function of medium or activity? An empirical pilot study. Journal of Behavioral Addictions, 3(1), 74-77. https://doi.org/10.1556/ jba.2.2013.016.

Guelmami, N., et al. (2021). Preliminary development of the social media disinformation scale (SMDS-12) and its association with social media addiction and mental health: COVID-19 as a pilot case study. JMIR Formative Research, 5(6), e27280. https://doi. org/10.2196/27280

Gür, E. F. (2020). "Technology" friend or foe in days of pandemic? Retrieved January 11, 2020, from https://yesilay.org.tr/tr/makal eler/pandemi-gunlerinde-teknoloji-dost-mu-dusman-mi

Hashemi, S. G. S., Hosseinnezhad, S., Dini, S., Griffiths, M. D., Lin, C. Y., \& Pakpour, A. H. (2020). The mediating effect of the cyberchondria and anxiety sensitivity in the association between problematic internet use, metacognition beliefs, and fear of COVID19 among Iranian online population. Heliyon, 6(10), e05135. https://doi.org/10.1016/j.heliyon.2020.e05135
Hayes, A. F. (2018). Introduction to mediation, moderation, and conditional process analysis: A regression-based approach. Guilford Press.

He, X., Zhang, Y., Chen, M., Zhang, J., Zou, W., \& Luo, Y. (2021). Media exposure to covid-19 predicted acute stress: A moderated mediation model of intolerance of uncertainty and perceived social support. Frontiers in Psychiatry, 11, 1552. https://doi.org/ 10.3389/fpsyt.2020.613368

Hu, L. T., \& Bentler, P. M. (1999). Cut off criteria for fit indices in covariance structure analysis: Conventional criteria versus new alternatives. Structural Equation Modeling, 6, 1-55. https://doi. org/10.1080/10705519909540118

Huang, Y., \& Zhao, N. (2020). Generalized anxiety disorder, depressive symptoms and sleep quality during COVID-19 outbreak in China: A web-based cross-sectional survey. Psychiatry Research, 288, 112954. https://doi.org/10.1016/j.psychres.2020.112954

Husnayain, A., Fuad, A., \& Su, E. C. Y. (2020). Applications of Google search trends for risk communication in infectious disease management: A case study of the COVID-19 outbreak in Taiwan. International Journal of Infectious Diseases, 95(6), 221-223. https://doi.org/10.1016/j.ijid.2020.03.021

Islam, M. S., Ferdous, M. Z., \& Potenza, M. N. (2020a). Panic and generalized anxiety during the COVID-19 pandemic among Bangladeshi people: An online pilot survey early in the outbreak. Journal of Affective Disorders, 276, 30-37. https://doi.org/10. 1016/j.jad.2020.06.049

Islam, M. S., et al. (2020b). Problematic internet use among young and adult population in Bangladesh: Correlates with lifestyle and online activities during the COVID-19 pandemic. Addictive Behaviors Reports, 12, 100311. https://doi.org/10.1016/j.abrep. 2020.100311

Ivanova, E. (2013). Internet addiction and cyberchondria-Their relationship with well-being. The Journal of Education, Culture, and Society, 4(1), 57-70. https://doi.org/10.15503/jecs20131-57-70

Ivanova, E., \& Karabeliova, S. (2014). Elaborating on Internet addiction and cyberchondria-relationships, direct and mediated effects. Journal of Education Culture and Society, 5(1), 127-144. https://doi.org/10.15503/jecs20141-127-144.

Jokic-Begic, N., Lauri Korajlija, A., \& Mikac, U. (2020). Cyberchondria in the age of COVID-19. PLoS ONE, 15(12), e0243704. https://doi.org/10.1371/journal.pone.0243704

Jungmann, S. M., \& Witthöft, M. (2020). Health anxiety, cyberchondria, and coping in the current COVID-19 pandemic: Which factors are related to coronavirus anxiety? Journal of Anxiety Disorders. https://doi.org/10.1016/j.janxdis.2020.102239

Jungmann, S. M., Brand, S., Kolb, J., \& Witthöft, M. (2020). Do Dr. Google and health apps have (comparable) side effects? An experimental study. Clinical Psychological Science, 8(2), 306317. https://doi.org/10.1177/2167702619894904

Kayis, A. R., Satici, B., Deniz, M. E., Satici, S. A., \& Griffiths, M. D. (2021). Fear of COVID-19, loneliness, smartphone addiction, and mental wellbeing among the Turkish general population: A serial mediation model. Behaviour \& Information Technology. https://doi.org/10.1080/0144929X.2021.1933181

Khazaal, Y., et al. (2020). Compulsive health-related internet use and cyberchondria. European Addiction Research. https://doi.org/10. $1159 / 000510922$

Kim, A., \& Dennis, A. R. (2019). Says who? The effects of presentation format and source rating on fake news in social media. MIS Quarterly, 43(3), 3. https://doi.org/10.25300/MISQ/2019/15188

King, A. L. S., Valença, A. M., Silva, A. C. O., Baczynski, T., Carvalho, M. R., \& Nardi, A. E. (2013). Nomophobia: Dependency on virtual environments or social phobia? Computers in Human Behavior, 29(1), 140-144. https://doi.org/10.1016/j.chb.2012. 07.025 
King, D. L., Delfabbro, P. H., Billieux, J., \& Potenza, M. N. (2020). Problematic online gaming and the COVID-19 pandemic. Journal of Behavioral Addictions, 9(2), 184-186. https://doi.org/10. 1556/2006.2020.00016

Kiraly, O., Potenza, M. N., Stein, D. J., King, D. L., Hodgins, D. C., Saunders, J. B., ... Demetrovics, Z. (2020). Preventing problematic internet use during the COVID-19 pandemic: Consensus guidance. Comprehensive Psychiatry, 100, 152180. https://doi. org/10.1016/j.comppsych.2020.152180

Kwon, M., Kim, D. J., Cho, H., \& Yang, S. (2013). The smartphone addiction scale: Development and validation of a short version for adolescents. PLoS ONE, 8(12), e83558. https://doi.org/10. 1371/journal.pone.0083558

Laato, S., Islam, A. N., Islam, M. N., \& Whelan, E. (2020a). What drives unverified information sharing and cyberchondria during the COVID-19 pandemic? European Journal of Information Systems, 29(39), 288-305. https://doi.org/10.1080/0960085X. 2020.1770632

Laato, S., Islam, A. N., Islam, M. N., \& Whelan, E. (2020b). What drives unverified information sharing and cyberchondria during the COVID-19 pandemic? European Journal of Information Systems. https://doi.org/10.1080/0960085X.2020.1770632

Lai, J., et al. (2020). Factors associated with mental health outcomes among health care workers exposed to coronavirus disease 2019. JAMA Network Open, 3(3), e203976. https://doi.org/10.1001/ jamanetworkopen.2020.3976

Lancet, T. (2020). COVID-19: Fighting panic with information. The Lancet, 395(10224), 537. https://doi.org/10.1016/S01406736(20)30379-2

Lau, J. T. F., Griffiths, S., Choi, K. C., \& Tsui, H. Y. (2010). Avoidance behaviors and negative psychological responses in the general population in the initial stage of the H1N1 pandemic in Hong Kong. BMC Infectious Diseases, 10, 139. https://doi.org/10. 1186/1471-2334-10-139

Lei, L., Huang, X., Zhang, S., Yang, J., Yang, L., \& Xu, M. (2020). Comparison of prevalence and associated factors of anxiety and depression among people affected by versus people unaffected by quarantine during the COVID-19 epidemic in Southwestern China. Medical Science Monitor, 26, e924609. https://doi.org/ 10.12659/MSM.924609

Li, S., Wang, Y., Xue, J., Zhao, N., \& Zhu, T. (2020). The impact of COVID-19 epidemic declaration on psychological consequences: A study on active Weibo users. International Journal of Environmental Research and Public Health, 17(6), 2032. https://doi. org/10.3390/ijerph17062032

Liu, Y., Gayle, A. A., Wilder-Smith, A., \& Rocklöv, J. (2020). The reproductive number of COVID-19 is higher compared to SARS coronavirus. Journal of Travel Medicine, 27(2), 1-4. https://doi. org/10.1093/jtm/taaa021

McElroy, E., \& Shevlin, M. (2014). The development and initial validation of the Cyberchondria Severity Scale (CSS). Journal of Anxiety Disorders, 28(2), 259-265. https://doi.org/10.1016/j. janxdis.2013.12.007

Main, A., Zhou, Q., Ma, Y., Luecken, L. J., \& Liu, X. (2011). Relations of SARS-related stressors and coping to Chinese college students' psychological adjustment during the 2003 Beijing SARS epidemic. Journal of Counseling Psychology, 58(3), 410-423. https://doi.org/10.1037/a0023632

Mattiuzzi, C., \& Lippi, G. (2020). Which lessons shall we learn from the 2019 novel coronavirus outbreak? Annals of Translational Medicine, 8(3), 48. https://doi.org/10.21037/atm.2020.02.06

McElroy, E., Kearney, M., Touhey, J., Evans, J., Cooke, Y., \& Shevlin, M. (2019). The CSS-12: Development and validation of a short-form version of the cyberchondria severity scale. Cyberpsychology, Behavior, and Social Networking, 22(5), 330-335. https://doi.org/10.1089/cyber.2018.0624
Milliyet Newspaper. (2020). Corona died by drinking disinfectant and ethyl alcohol to protect against the virüs. Retrieved January 11, 2020, from https://www.milliyet.com.tr/gundem/ corona-virusten-korunmak-icin-dezenfektan-ve-etil-alkolicince-oldu-6199258

Norr, A. M., Allan, N. P., Boffa, J. W., Raines, A. M., \& Schmidt, N. B. (2015). Validation of the Cyberchondria Severity Scale (CSS): Replication and extension with bifactor modeling. Journal of Anxiety Disorders, 31, 58-64. https://doi.org/10. 1016/j.janxdis.2015.02.001

Noyan, C. O., Enez-Darcin, A., Nurmedov, S., Yilmaz, O., \& Dilbaz, N. (2015). Validity and reliability of the Turkish version of the Smartphone Addiction Scale-Short version among university students. Anatolian Journal of Psychiatry, 16(1), 73-81. https://doi.org/10.5455/apd.176101

Oosterhoff, B., Palmer, C. A., Wilson, J., \& Shook, N. (2020). Adolescents' motivations to engage in social distancing during the COVID-19 pandemic: associations with mental and social health. Journal of Adolescent Health, 67(2), 179-185. https:// doi.org/10.1016/j.jadohealth.2020.05.004

Pakpour, A. H., \& Griffiths, M. D. (2020). The fear of COVID-19 and its role in preventive behaviors. Journal of Concurrent Disorders. http://irep.ntu.ac.uk/id/eprint/39561/

Peng, E.Y.-C., et al. (2010). Population-based post-crisis psychological distress: An example from the SARS outbreak in Taiwan. Journal of the Formosan Medical Association, 109(7), 524-532. https://doi.org/10.1016/S0929-6646(10)60087-3

Qiu, J., Shen, B., Zhao, M., Wang, Z., Xie, B., \& Xu, Y. (2020). A nationwide survey of psychological distress among Chinese people in the COVID-19 epidemic: Implications and policy recommendations. General Psychiatry, 33(2), e100213. https://doi.org/ 10.1136/gpsych-2020-100213

Rajkumar, R. P. (2020). COVID-19 and mental health: A review of the existing literature. Asian Journal of Psychiatry, 52, 102066. https://doi.org/10.1016/j.ajp.2020.102066

Roy, D., Tripathy, S., Kar, S. K., Sharma, N., Verma, S. K., \& Kaushal, V. (2020). Study of knowledge, attitude, anxiety \& perceived mental healthcare need in Indian population during COVID-19 pandemic. Asian Journal of Psychiatry, 51, 102083. https://doi. org/10.1016/j.ajp.2020.102083

Rovetta, A., \& Bhagavathula, A. S. (2020). Novel coronavirus (COVID19)-related web search behavior and infodemic attitude in Italy: Infodemiological study. JMIR Public Health and Surveillance, 6(2), e19374. https://doi.org/10.2196/19374

Saadatian-Elahi, M., Facy, F., Del Signore, C., \& Vanhems, P. (2010). Perception of epidemic's related anxiety in the general French population: A cross-sectional study in the Rhône-Alpes region. BMC Public Health, 10, 191. https://doi.org/10.1186/ 1471-2458-10-191

Sabah Newspaper. (2020). Last minute: Incredible event! They drank disinfectant and died. Retrieved January 11, 2020, from https:// www.sabah.com.tr/dunya/2020/06/28/son-dakika-akilalmazolay-dezenfekten-icip-olduler

Satici, B., Gocet-Tekin, E., Deniz, M. E., \& Satici, S. A. (2020). Adaptation of the Fear of COVID-19 Scale: Its association with psychological distress and life satisfaction in Turkey. International Journal of Mental Health and Addiction. https://doi.org/10.1007/ s11469-020-00294-0

Schimmenti, A., Billieux, J., \& Starcevic, V. (2020). The four hoursemen of fear: An integrated model of understanding fear experiences during the COVID-19 pandemic. Clinical Neuropsychiatry, 17(2), 41-45. https://doi.org/10.36131/CN20200202

Selvi, Y., Turan, S. G., Sayin, A. A., Boysan, M., \& Kandeger, A. (2018). The Cyberchondria Severity Scale (CSS): Validity and reliability study of the Turkish version. Sleep and Hypnosis, 
20(4), 241-246. https://doi.org/10.5350/Sleep.Hypn.2018.20. 0157

Servidio, R., Bartolo, M. G., Palermiti, A. L., \& Costabile, A. (2021). Fear of COVID-19, depression, anxiety, and their association with Internet addiction disorder in a sample of Italian students. Journal of Affective Disorders Reports, 4, 100097. https://doi. org/10.1016/j.jadr.2021.100097

Sha, P., Sariyska, R., Riedl, R., Lachmann, B., \& Montag, C. (2019). Linking internet communication and smartphone use disorder by taking a closer look at the Facebook and WhatsApp applications. Addictive Behaviors Reports, 9, 100148. https://doi.org/ 10.1016/j.abrep.2018.100148

Soni, R., Upadhyay, R., \& Jain, M. (2017). Prevalence of smart phone addiction, sleep quality and associated behaviour problems in adolescents. International Journal of Research in Medical Sciences, 5(2), 515-519.

Starcevic, V., \& Berle, D. (2013). Cyberchondria: Towards a better understanding of excessive health-related Internet use. Expert Review of Neurotherapeutics, 13(2), 205-213. https://doi.org/ 10.1586/ern.12.162

Starcevic, V. (2017). Cyberchondria: Challenges of problematic online searches for health-related information. Psychotherapy and Psychosomatics, 86(3), 129-133. https://doi.org/10.1159/000465525

Starcevic, V., Schimmenti, A., Billieux, J., \& Berle, D. (2021). Cyberchondria in the time of the COVID-19 pandemic. Human Behavior and Emerging Technologies, 3(1), 53-62. https://doi.org/10. 1002/hbe2.233

van Deursen, A. J. (2020). Digital inequality during a pandemic: Quantitative study of differences in COVID-19-related internet uses and outcomes among the general population. Journal of Medical Internet Research, 22(8), e20073. https://doi.org/10.2196/20073

Vismara, M., Caricasole, V., Starcevic, V., Cinosi, E., Dell'Osso, B., Martinotti, G., \& Fineberg, N. A. (2020). Is cyberchondria a new transdiagnostic digital compulsive syndrome? A systematic review of the evidence. Comprehensive Psychiatry, 99(2020), 152167. https://doi.org/10.1016/j.comppsych.2020.152167

We Are Social. (2020a). Digital in 2020. Retrieved from https://weare social.com/digital-2020

We Are Social. (2020b). Digital 2020: Turkey. Retrieved from https:// datareportal.com/reports/digital-2020-turkey

White, R. W., \& Horvitz, E. (2009). Cyberchondria: Studies of the escalation of medical concerns in web search. ACM Transactions on Information Systems (TOIS), 27(4), 1-37. https://doi.org/10. 1145/1629096.1629101.

Wang, C., Pan, R., Wan, X., Tan, Y., Xu, L., Ho, C. S., \& Ho, R. C. (2020). Immediate psychological responses and associated factors during the initial stage of the 2019 coronavirus disease (COVID-19) epidemic among the general population in China. International Journal of Environmental Research and Public Health, 17(5), 1729. https://doi.org/10.3390/ijerph17051729

Wilder-Smith, A., \& Freedman, D. O. (2020). Isolation, quarantine, social distancing and community containment: Pivotal role for old-style public health measures in the novel coronavirus (2019nCoV) outbreak. Journal of Travel Medicine, 27(2), taa020. https://doi.org/10.1093/jtm/taaa020

World Health Organization. (2019). Rolling updates on coronavirus disease (COVID-19). Retrieved January 11, 2020, froms https:// www.who.int/emergencies/diseases/novel-coronavirus-2019/ events-as-they-happen
World Health Organization. (2020a). Q\&A on coronaviruses (COVID19). Retrieved January 11, 2020, from https://www.who.int/ news-room/q-a-detail/q-a-coronaviruses\#: :text=symptoms

World Health Organization. (2020b). WHO Director-General's opening remarks at the media briefing on COVID-19-18 March 2020. Retrieved January 11, 2020, from https://www.who.int/dg/speec hes/detail/who-director-general-s-opening-remarks-at-the-mediabriefing-on-covid-19---18-march-2020

World Health Organization. (2020c). Report of the WHO-China joint mission on coronavirus disease 2019 (COVID-19). Retrieved January 11, 2020, from https://www.who.int/docs/default-source/ coronaviruse/who-china-joint-mission-on-covid-19-final-report. pdf

Wu, X., Nazari, N., \& Griffiths, M. D. (2021). Using fear and anxiety related to COVID-19 to predict cyberchondria: Cross-sectional survey study. Journal of Medical Internet Research, 23(6), e26285. https://doi.org/10.2196/26285

Xiang, Y. T., Yang, Y., Li, W., Zhang, L., Zhang, Q., Cheung, T., \& $\mathrm{Ng}$, C. H. (2020). Timely mental health care for the 2019 novel coronavirus outbreak is urgently needed. The Lancet Psychiatry, 7(3), 228-229. https://doi.org/10.1016/S2215-0366(20)30046-8

Xiao, H., Zhang, Y., Kong, D., Li, S., \& Yang, N. (2020). The effects of social support on sleep quality of medical staff treating patients with coronavirus disease 2019 (COVID-19) in January and February 2020 in China. Medical Science Monitor, 26, e923549. https://doi.org/10.12659/MSM.923549

Xie, X.-F., Stone, E., Zheng, R., \& Zhang, R.-G. (2011). The "Typhoon Eye Effect": Determinants of distress during the SARS epidemic. Journal of Risk Research, 14(9), 1091-1107. https://doi.org/10. 1080/13669877.2011.571790

Yang, J., Fu, X., Liao, X., \& Li, Y. (2020). Association of problematic smartphone use with poor sleep quality, depression, and anxiety: A systematic review and meta-analysis. Psychiatry Research, 284, 112686. https://doi.org/10.1016/j.psychres.2019.112686

Yılmaz, G., Şar, A. H., \& Civan, S. (2015). Investigation of adolescent mobile phone addiction by social anxiety effect of some variable. Online Journal of Technology Addiction \& Cyberbullying, 2(2), 20-37.

Yu, M., Li, Z., Yu, Z., He, J., \& Zhou, J. (2020). Communication related health crisis on social media: A case of COVID-19 outbreak. Current Issues in Tourism. https://doi.org/10.1080/13683 500.2020 .1752632

Zhang, Y., \& Ma, Z. F. (2020). Impact of the COVID-19 pandemic on mental health and quality of life among local residents in Liaoning Province, China: A cross-sectional study. International Journal of Environmental Research and Public Health, 17(7), 1-12. https://doi.org/10.3390/ijerph17072381

Zheng, H., Sin, S. C. J., Kim, H. K., \& Theng, Y. L. (2020). Cyberchondria: A systematic review. Internet Research. https://doi.org/ 10.1108/INTR-03-2020-0148

Publisher's note Springer Nature remains neutral with regard to jurisdictional claims in published maps and institutional affiliations. 